\title{
Radiation May Indirectly Impair Growth Resulting in Reduced Standing Height via Subclinical Inflammation in Atomic-Bomb Survivors Exposed at Young Ages
}

\author{
Eiji Nakashima, ${ }^{1}$ Kazuo Neriishi, ${ }^{2}$ and Wan-Ling $\mathrm{Hsu}^{1}$ \\ ${ }^{1}$ Department of Statistics, Radiation Effects Research Foundation, Hijiyama Park 5-2, Minami-ku, Hiroshima 732-0815, Japan \\ ${ }^{2}$ Department of Clinical Studies, Radiation Effects Research Foundation, Hijiyama Park 5-2, Minami-ku, Hiroshima 732-0815, Japan \\ Correspondence should be addressed to Eiji Nakashima; nakasima@rerf.or.jp
}

Received 23 October 2014; Revised 16 January 2015; Accepted 16 January 2015

Academic Editor: Leo J. Schouten

Copyright (C) 2015 Eiji Nakashima et al. This is an open access article distributed under the Creative Commons Attribution License, which permits unrestricted use, distribution, and reproduction in any medium, provided the original work is properly cited.

For young atomic-bomb (A-bomb) survivors, A-bomb radiation's (total) effect on standing height is thought to comprise the sum of direct effect and indirect effect via inflammation. With the data of five inflammatory markers-white blood cell count, sialic acid, corrected erythrocyte sedimentation rate (ESR), $\alpha 1$ globulin, and $\alpha 2$ globulin-obtained in adulthood during the period 1988 to 1992, a summary inflammatory index was constructed as a surrogate for the five subclinical inflammatory markers. For 3,327 A-bomb survivors exposed at ages of less than 25 years, a structural equation model was analyzed to measure direct radiation effects on adult height as well as mediating effect of radiation via inflammation on the height after adjustment for other risk factors, smoking, cancer, inflammatory disease, obesity, and diabetes mellitus. The mediation proportion of the radiation effect on height via inflammation was approximately $5 \%$ for both sexes for all ages, and indirect dose effects via inflammation were statistically significant for both sexes combined and for females exposed at ages 0 to 5 years. Indirect dose effects for all ages via sialic acid, corrected ESR, and $\alpha 2$ globulin were marginally significant for both sexes combined and for females. These proportions are likely underestimated.

\section{Introduction}

It is known that childhood exposure to radiation leads to reduced growth in terms of standing height among the atomic-bomb (A-bomb) survivors $[1,2]$. Earlier crosssectional studies indicated that radiation exposure also resulted in higher white blood cell counts $[3,4]$. In a special study from 1988 to 1992, such subclinical inflammatory markers as white blood cell count, sialic acid, erythrocyte sedimentation rate, $\alpha 1$ globulin, and $\alpha 2$ globulin were shown to increase with radiation dose in A-bomb survivors [5]. Subclinical inflammation may persist over a long period, as reported in a longitudinal study on trend in white blood cell count among A-bomb survivors [6].

Children with inflammatory disease were shown to have growth impairment in a human study [7] and the same phenomenon was also reported in a mouse study [8]. Experiments indicated that inflammatory cytokines [9] inhibit growth hormone and insulin-like growth factor-1 (IGF-1), while IGF-1 was shown to be an important growth factor in animal experiments $[10,11]$. Those findings imply the workable hypothesis that persistent subclinical inflammation may result in growth impairment due to inhibition of growth hormone and IGF-1 for people exposed to A-bomb radiation in childhood.

Neriishi et al. [12] discussed the role of inflammation in relation to radiation dose and height in the A-bomb survivors. In a report based on data obtained during the period from 1988 to 1992, a principal component analysis [13] was conducted to construct the first principal component score (size factor) with all positive coefficients as a summary measure of subclinical inflammation derived from the five inflammatory markers white blood cell count, sialic acid, corrected erythrocyte sedimentation rate, $\alpha 1$ globulin, and $\alpha 2$ globulin. The report discussed the relationship among height, radiation dose, and subclinical inflammatory index. 
The authors inferred that there exists an indirect inflammation effect on height from radiation dose via subclinical inflammation in which mediation proportions [14] of the radiation dose effect on height via inflammation index constructed on the basis of the principal component analysis were $14.1 \%$ for males, $6.1 \%$ for females, and $7.4 \%$ for both sexes combined for all ages. They also reported that the mediation proportion of radiation dose effect via inflammation was larger for females and for those with younger age at exposure. However, in their analysis, the authors did not adjust the inflammatory index for other risk factors such as smoking, cancer, inflammatory disease, obesity, and diabetes mellitus [15].

In this paper, in addition to the direct effect of A-bomb radiation, we show that there exists an indirect mediation effect of radiation on height via the process of subclinical inflammation. To characterize the indirect mediation effect, we applied a structural equation model (SEM) [16] with the three measurements in young A-bomb survivors of adult standing height, radiation dose, and subclinical inflammatory index. In this SEM analysis, the subclinical inflammatory index was constructed by factor analysis using the five inflammatory markers white blood cell count, sialic acid, corrected erythrocyte sedimentation rate, $\alpha 1$ globulin, and $\alpha 2$ globulin. The inflammatory index was used as an indirect mediation variable on height, where the inflammatory index was adjusted for such risk factors as smoking, cancer, inflammatory disease, body mass index, and diabetes mellitus. Based on the assumption that subclinical inflammation observed in adulthood may reflect persistent inflammation arising from radiation exposure in childhood, we infer a mediation effect of radiation via inflammation to be evidence of such mediation at the time of exposure.

\section{Materials and Statistical Methods}

2.1. Materials. The Adult Health Study (AHS) cohort $[17,18]$ was established in 1958, with a subsequent revision in 1977 by the Radiation Effects Research Foundation (RERF), formerly the Atomic Bomb Casualty Commission (ABCC), and included about 17,400 people exposed to A-bomb radiation in Hiroshima and Nagasaki. The AHS subjects were invited to undergo biennial health examinations at ABCC/RERF clinics starting in 1958, and the relevant data were recorded in the AHS database. We refer to each of these two-year periods as an "examination cycle." The original AHS sample consisted of about 15,000 exposed people drawn from the Life Span Study (LSS) cohort [19], with the core AHS group numbering about 5,000 survivors who were exposed at distance of less than $2 \mathrm{~km}$ from either of the hypocenters and reported signs and symptoms of acute radiation syndrome. Two counterparts to this core group of subjects, one for those exposed less than $2 \mathrm{~km}$ from either of the hypocenters without any signs nor symptoms of acute radiation syndrome and one for those exposed at distance of greater than $3 \mathrm{~km}$ from either of the hypocenters, were also included in the AHS. These two counterparts were city-, age-, and sex-matched samples drawn from the LSS cohort, each similar in size to the core group. In 1977, about 2,400 additional exposed persons from the LSS were added to the AHS to intentionally enrich the number of higher-dose persons. About $63 \%$ of the AHS subjects were exposed within $2 \mathrm{~km}$ of the hypocenter. Almost all survivors exposed less than $2 \mathrm{~km}$ from hypocenter are included in the AHS cohort.

Dose-response analyses were based on the 2002 Dosimetry System (DS02) [20] weighted bone marrow radiation dose in gray equivalents (Gy), which is the sum of bone marrow gamma radiation dose plus 10 times the bone marrow neutron radiation dose. Gamma and neutron bone marrow doses were adjusted for log-normal dose error with 35\% coefficient of variation after truncation at $4 \mathrm{~Gy}$ for shielded total kerma dose [21], which reduces estimation bias for radiation effects in regression analysis.

A special cross-sectional study [5] on subclinical inflammation in the AHS was conducted between July 1, 1988, and June 30,1992. A total of 4,076 AHS subjects with estimated radiation dose and who were in the cities at the time of bombings (ATB) participated in the AHS health examinations, with ages ATB ranging from 0 to less than 25 years excluding those exposed in utero. The five inflammatory markers, white blood cell (WBC) count $\left(\times 10^{2} / \mathrm{mm}^{3}\right)$, sialic acid (SA) $(\mathrm{mg} / \mathrm{dL})$, corrected erythrocyte sedimentation rate (ESR) ( $\mathrm{mm} /$ hour), $\alpha 1$ globulin ( $\alpha$ lglob) (\%), and $\alpha 2$ globulin ( $\alpha 2 \mathrm{glob})(\%)$ were measured. In addition to the measurements of these five markers of inflammation, corresponding disease information in the period from 1988 to 1992, such as that obtained from medical records regarding cancer, inflammatory diseases (Table 2), and diabetes mellitus, was included. Information on smoking from questionnaire was also collected during the 1988-1992 health examinations, with such information also available in the AHS database. The first measurement among routinely recorded standing height and body weights at ages ranging from 25 to 45 years was selected to obtain a mature or adult standing height for the subjects. In this age period, body mass index (BMI) would be an important surrogate for socioeconomic or genetic effects on height other than radiation dose. After excluding subjects with missing information on inflammatory markers (134 subjects), cancer (2 subjects), inflammatory disease (2 subjects), diabetes (2 subjects), smoking (22 subjects), and standing height (634 subjects) or body weight (637 subjects), 3,327 subjects were selected for the analysis (Table 1 ). The missing data is approximately $19 \%$ in the original 4,076 AHS study sample.

2.2. Statistical Methods. For the radiation effect on standing height in relation to inflammation, we applied a structural equation model (SEM) [16] to the data using the maximum likelihood method. A latent inflammatory index was derived using confirmatory factor analysis with the five inflammatory markers, and two regression analyses were conducted for height and the inflammatory index produced with the aforementioned confirmatory factor analysis. Figure 1 shows the hypothesized relationship among radiation, inflammation, and height for this SEM model with three models M1, M2, and M3 fit simultaneously using the M-plus program $[14,16]$. 
TABLE 1: Data profile of 3,327 atomic bomb survivors included in analysis.

\begin{tabular}{|c|c|c|c|}
\hline \multirow{2}{*}{ Variables } & \multicolumn{3}{|c|}{ Sex } \\
\hline & Male $(1,143)$ & Female $(2,184)$ & Both sexes $(3,327)$ \\
\hline Hiroshima (\%) & $714(62.5)$ & $1430(65.5)$ & $2144(64.4)$ \\
\hline Age at exposure $(y r)^{(a)}$ & $12.6(0,25.0)$ & $14.9(0.1,25.0)$ & $14.1(0,25.0)$ \\
\hline Bone marrow dose $(\mathrm{Gy})^{(\mathrm{a})}$ & $0.533(0,4.12)$ & $0.433(0,4.42)$ & $0.468(0,4.42)$ \\
\hline Standing height $(\mathrm{cm})^{(\mathrm{b})}$ & $162.7(6.13)$ & $151.1(5.39)$ & $155.1(7.90)$ \\
\hline Weight $(\mathrm{kg})^{(\mathrm{b})}$ & $54.8(7.51)$ & $48.8(7.11)$ & $50.9(7.79)$ \\
\hline BMI $\left(\mathrm{kg} / \mathrm{m}^{2}\right)^{(\mathrm{b})}$ & $20.7(2.42)$ & $21.3(2.83)$ & $21.1(2.71)$ \\
\hline Smoker (\%) & $617(54.0)$ & $207(9.5)$ & $824(24.8)$ \\
\hline Cancer (\%) & $56(4.9)$ & $182(8.3)$ & $238(7.2)$ \\
\hline Inflammatory diseases (\%) & $491(43.0)$ & $922(42.2)$ & $1413(42.5)$ \\
\hline Diabetes mellitus (\%) & $133(11.6)$ & $155(7.1)$ & $288(8.7)$ \\
\hline $\mathrm{WBC}\left(\times 10^{2} / \mathrm{mm}^{3}\right)^{(\mathrm{b})}$ & $66.7(18.72)$ & $55.7(14.75)$ & $59.5(17.03)$ \\
\hline Sialic Acid (mg/dL) ${ }^{(b)}$ & $68.6(9.20)$ & $67.9(9.22)$ & $68.2(9.22)$ \\
\hline $\operatorname{ESR}(\mathrm{mm} / \text { hour })^{(\mathrm{b})}$ & $11.4(8.15)$ & $20.0(9.72)$ & $17.1(10.07)$ \\
\hline$\alpha 1$ globulin $(\%)^{(\mathrm{b})}$ & $3.4(0.72)$ & $3.3(0.66)$ & $3.3(0.68)$ \\
\hline$\alpha 2$ globulin $(\%)^{(\mathrm{b})}$ & $9.4(1.38)$ & $9.6(1.35)$ & $9.5(1.37)$ \\
\hline
\end{tabular}

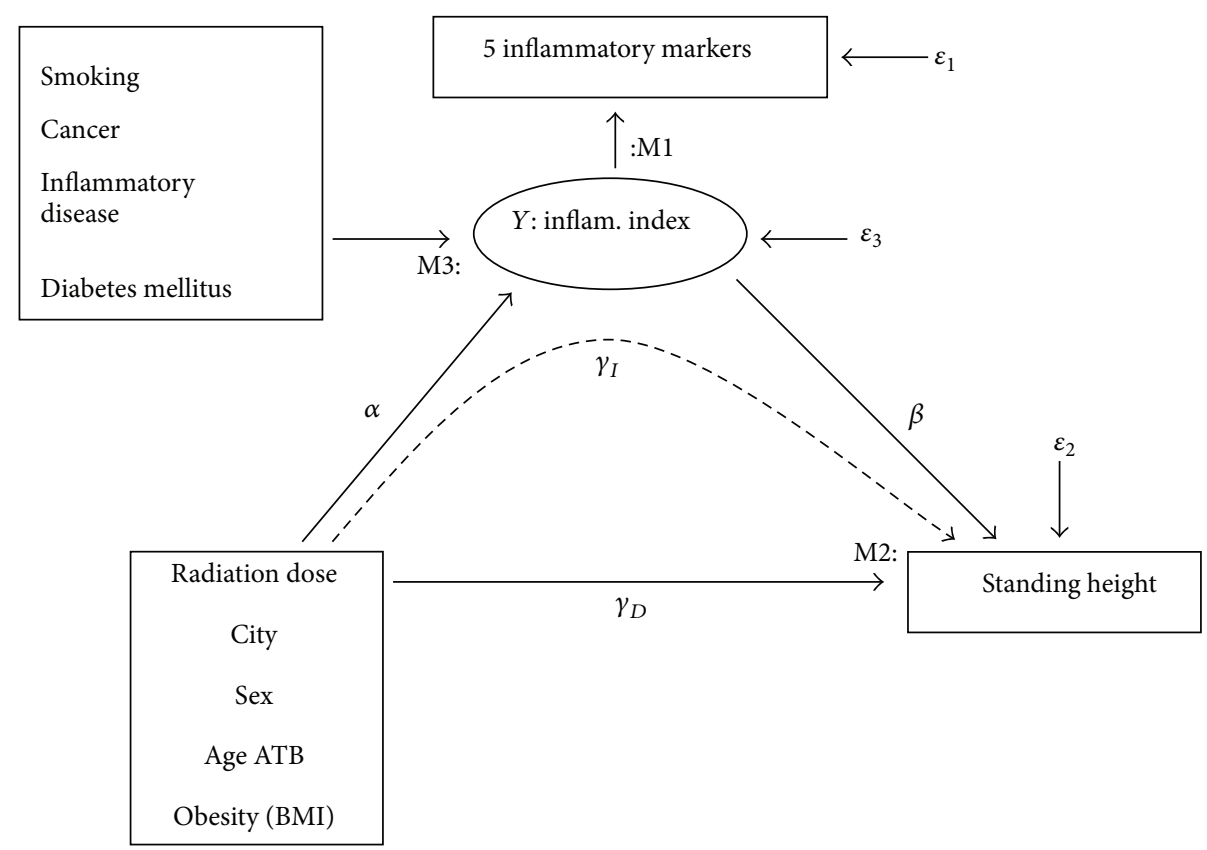

FIGURE 1: Path diagram of the structural equation model (SEM) consisting of models M1, M2, and M3. Variable $Y$ is the latent unobserved inflammatory index from the inflammatory markers obtained in the period from 1988 to 1992 with model M1. Effect $\alpha$ is the radiation dose effect on the inflammatory index in model M3, effect $\beta$ is the inflammatory index effect on standing height in model M2, and effect $\gamma_{D}$ is the direct radiation dose effect on standing height in model M2. Estimate $\gamma_{I}=\alpha \beta$ is the indirect radiation dose effect on standing height via inflammation. Error vector $\varepsilon_{1}$ and scalar error variables $\varepsilon_{2}$ and $\varepsilon_{3}$ are mutually independent normal random errors with zero means for models M1, M2, and M3, respectively. Variables shown in ovals are latent variables and variables in rectangles are observed variables.

M1: confirmatory factor analysis (CFA) with the maximum likelihood method to derive a latent unobserved inflammatory surrogate vector $Y$ as a linear combination of the five inflammatory markers, that is, WBC, SA, ESR, $\alpha$ lglob, and $\alpha 2 \mathrm{glob}$, for each subject. The factor loading for SA, which has the largest mean and the minimum coefficient of variation among the five markers, was set to one throughout the analysis. In mathematical expression, let $x$ be a 5 by $N$ observation matrix consisting of five inflammatory markers, WBC, SA, ESR, $\alpha$ lglob, and $\alpha 2$ glob, from each of $N$ subjects, 
TABLE 2: Details of the inflammatory disease cases among 3,327 subjects found from 1988 to 1992.

\begin{tabular}{|c|c|c|}
\hline Disease & $\begin{array}{c}\text { Number of } \\
\text { cases }\end{array}$ & Percentage \\
\hline Inflammatory diseases & 1,413 & $100 \%$ \\
\hline Infections & 425 & 30.1 \\
\hline HIV & 0 & 0 \\
\hline Thyroiditis & 137 & 9.7 \\
\hline Tonsillitis & 5 & 0.4 \\
\hline Pneumonia & 19 & 1.3 \\
\hline Influenza & 0 & 0 \\
\hline Bronchitis & 16 & 1.1 \\
\hline Crohn's disease & 0 & 0 \\
\hline Ulcerative colitis & 0 & 0 \\
\hline Chronic liver disease (cirrhosis) & 446 & 31.6 \\
\hline Chronic pancreatitis & 15 & 1.1 \\
\hline Pancreatitis & 24 & 1.7 \\
\hline Acute glomerulonephritis & 0 & 0 \\
\hline Subacute nephrosis & 1 & 0.1 \\
\hline Chronic glomerulonephritis & 11 & 0.8 \\
\hline Glomerulonephritis & 1 & 0.1 \\
\hline SLE (systemic lupus erythematosus) & 4 & 0.3 \\
\hline PSS (progressive systemic sclerosis) & 2 & 0.1 \\
\hline Sjogren's syndrome & 1 & 0.1 \\
\hline Dermatomyositis & 0 & 0 \\
\hline Polymyositis & 1 & 0.1 \\
\hline Rheumatoid arthritis & 40 & 2.8 \\
\hline Osteoarthritis & 543 & 38.4 \\
\hline
\end{tabular}

Note that the total number of cases in all subcategories is greater than the total number of cases, 1413, because subjects could have multiple inflammatory diseases.

where the number of subjects $N$ is 3,327 for both sexes, 1,143 for males and 2,184 for females, and $\mu$ is the 5 by $N$ mean matrix of the observation matrix $x$. Then we make a factor analysis M1: $x-\mu=L Y+\varepsilon$, where $L$ is the 5 by one factor loading matrix and $Y$ is a single one by $N$ factor score vector. We assume the factor score vector $Y$ and the error $\varepsilon$ are independent and the expectation of $Y$ is zero. For the estimation of $L$ and $Y$ in CFA, the maximum likelihood method will be used in M-plus software.

M2: regression analysis of height on city, sex, age ATB, bone marrow radiation dose, body mass index, and inflammatory index $Y$ using the main effects model, where body mass index was included in the model as (BMI-20)/10, where body mass index $\left(\mathrm{BMI}\left(\mathrm{kg} / \mathrm{m}^{2}\right)\right)$ was calculated as the body weight $(\mathrm{kg})$ divided by square of the standing height $(\mathrm{m})$. The inclusion of adult BMI at ages $25 \mathrm{y}$ to $45 \mathrm{y}$ in the regression of height could exclude the socioeconomic effect on the survivors other than the pure biological effect of radiation dose. We have no such index other than BMI that reflects the socioeconomic status.
M3: regression analysis of inflammatory index $Y$ on city, sex, age ATB, bone marrow radiation dose, body mass index, smoking indicator, cancer indicator, inflammatory disease indicator, and diabetes indicator using the main effects model, where body mass index was included in the model as $(\mathrm{BMI}-20) / 10$. The radiation dose effect was adjusted by inflammatory variables such as smoking, cancer, inflammatory diseases, diabetes and obesity with this regression.

In Figure 1, the confirmatory factor analysis M1 was conducted for the five inflammatory markers to find the inflammatory index source of the five inflammatory markers. In the linear regression M2 of height, as shown in Figure 1, the regression coefficient for direct dose effect on height is denoted as $\gamma_{D}$ and the regression coefficient of inflammatory index on height as $\beta$. In the linear regression M3 of inflammatory index, the regression coefficient of radiation dose is $\alpha$. The total radiation effect on height, $\gamma_{T}$, is the sum of direct radiation effect $\left(\gamma_{D}\right)$ plus indirect radiation effect $\left(\gamma_{I}\right)$, $\gamma_{T}=\gamma_{D}+\gamma_{I}$, where the indirect radiation effect on height via inflammation, $\gamma_{I}$, is the product of $\alpha$ and $\beta$; that is, $\gamma_{I}=\alpha \beta$. The mediation proportion (MP) [14] of radiation dose via inflammation was defined as MP $=\gamma_{I} / \gamma_{T}=\gamma_{I} /\left(\gamma_{D}+\gamma_{I}\right)$, which is unit-free and estimated by replacing the indirect and direct effects with their estimates $\widehat{\gamma}_{I}=\widehat{\alpha} \widehat{\beta}$ and $\widehat{\gamma}_{D}$, respectively.

The bootstrap standard errors (SEs) of the parameter estimates with 1,000 resamplings were obtained for the indirect, direct, and total dose effects during SEM fitting of models M1, M2, and M3 with the M-plus program [16]. Because the bootstrap standard error is not available for MP, the first order approximate standard error for the estimate of MP is calculated as $\widehat{\sigma}_{\mathrm{MP}}=\sqrt{\left[\widehat{\sigma}_{T}^{2}\left(\widehat{\gamma}_{I} / \widehat{\gamma}_{T}\right)^{2}+\widehat{\sigma}_{I}^{2}\right] / \widehat{\gamma}_{T}^{2}}$ neglecting covariance between total and indirect effects due to nonavailability in the output of M-plus program, where $\widehat{\sigma}_{T}$ and $\widehat{\sigma}_{I}$ are standard errors for total effect $\widehat{\gamma}_{T}$ and indirect effect $\widehat{\gamma}_{I}$, respectively, and the approximate $95 \%$ confidence interval of the MP was constructed using this standard error $\widehat{\sigma}_{\mathrm{MP}}$.

Because human growth depends on sex and age, the analyses were also conducted by sex and by age ATB groups: ages $0-5,6-17,0-17$, and 18-24 years ATB. The choice of the cutoff ages of 6 and 18 years for ATB groups was somewhat arbitrary. It was assumed that the cutoff ages for the first growth period had little sex difference in growth, with the second growth period having appreciable sex difference in growth, and the maturation period with very little growth. By inflammatory markers the SEM analysis using each of the five inflammatory markers instead of the inflammatory index from M1 was also conducted for all subjects.

\section{Results}

Table 1 shows the data profile of the 3,327 subjects undergoing analysis. Sixty-six percent of the subjects were female and 64 percent of the subjects were in Hiroshima. The mean bone marrow radiation dose was $0.468 \mathrm{~Gy}$. Smoking rates were $54 \%$ in males and $10 \%$ for females. We observed a higher percentage of cancer cases in females (8.3\%) and a higher percentage of diabetes among men (11.6\%). The percentage 
TABLE 3: Results of structural equation model (SEM: M1, M2, and M3) analysis with all ages (0-<25 years) for both sexes combined and by sex. Intercepts were excluded.

\begin{tabular}{|c|c|c|c|c|c|c|c|c|c|}
\hline \multirow{3}{*}{ Variable } & \multicolumn{9}{|c|}{ Factor loading in CFA: $\mathrm{M1}^{+}$} \\
\hline & \multicolumn{3}{|c|}{ Both sexes $(n=3327)$} & \multicolumn{3}{|c|}{ Males $(n=1143)$} & \multicolumn{3}{|c|}{ Females $(n=2184)$} \\
\hline & Estimate & SE & $P^{*}$ & Estimate & SE & $P^{*}$ & Estimate & SE & $P^{*}$ \\
\hline WBC & 0.642 & 0.045 & $<0.001$ & 0.774 & 0.092 & $<0.001$ & 0.574 & 0.055 & $<0.001$ \\
\hline SA & 1.000 & 0.000 & - & 1.000 & 0.000 & - & 1.000 & 0.000 & - \\
\hline ESR & 0.651 & 0.030 & $<0.001$ & 0.566 & 0.028 & $<0.001$ & 0.703 & 0.035 & $<0.001$ \\
\hline$\alpha 1$ glob & 0.029 & 0.002 & $<0.001$ & 0.035 & 0.003 & $<0.001$ & 0.026 & 0.002 & $<0.001$ \\
\hline$\alpha 2$ glob & 0.111 & 0.005 & $<0.001$ & 0.119 & 0.007 & $<0.001$ & 0.103 & 0.005 & $<0.001$ \\
\hline \multicolumn{10}{|c|}{ Linear regression analysis of standing height: $\mathrm{M} 2^{++}$} \\
\hline City & -0.584 & 0.205 & 0.004 & -0.063 & 0.353 & 0.858 & -0.879 & 0.240 & $<0.001$ \\
\hline Sex & -11.25 & 0.218 & $<0.001$ & - & - & - & - & - & - \\
\hline $\mathrm{ATB} / 10$ & -1.482 & 0.153 & $<0.001$ & -2.090 & 0.287 & $<0.001$ & -1.201 & 0.181 & $<0.001$ \\
\hline Dose & -0.663 & 0.133 & $<0.001$ & -0.622 & 0.221 & 0.005 & -0.696 & 0.165 & $<0.001$ \\
\hline $\mathrm{BMI} / 10$ & -1.321 & 0.381 & 0.001 & -1.564 & 0.820 & 0.056 & -1.295 & 0.441 & 0.003 \\
\hline Infl. index & -0.035 & 0.012 & 0.004 & -0.027 & 0.023 & 0.243 & -0.037 & 0.013 & 0.006 \\
\hline$R^{2}$ & 0.509 & & & 0.051 & & & 0.044 & & \\
\hline \multicolumn{10}{|c|}{ Linear regression analysis of inflammatory index: $\mathrm{M3}^{++}$} \\
\hline City & -1.347 & 0.329 & $<0.001$ & -2.063 & 0.540 & $<0.001$ & -0.890 & 0.429 & 0.038 \\
\hline Sex & 0.859 & 0.491 & 0.080 & - & - & - & - & - & - \\
\hline ATB/10 & 2.326 & 0.258 & $<0.001$ & 2.213 & 0.403 & $<0.001$ & 2.293 & 0.325 & $<0.001$ \\
\hline Dose & 1.086 & 0.214 & $<0.001$ & 1.311 & 0.317 & $<0.001$ & 0.858 & 0.279 & 0.002 \\
\hline BMI/10 & 2.100 & 0.598 & $<0.001$ & 0.126 & 1.083 & 0.907 & 2.995 & 0.723 & $<0.001$ \\
\hline Cancer & 0.338 & 0.694 & 0.626 & -1.441 & 1.420 & 0.310 & 0.954 & 0.843 & 0.258 \\
\hline Infl. dis. & 0.562 & 0.311 & 0.071 & 0.942 & 0.515 & 0.068 & 0.395 & 0.392 & 0.314 \\
\hline $\mathrm{DM}$ & 2.652 & 0.675 & $<0.001$ & 0.710 & 0.828 & 0.391 & 4.243 & 1.078 & $<0.001$ \\
\hline SMK & 2.880 & 0.461 & $<0.001$ & 3.403 & 0.520 & $<0.001$ & 1.957 & 0.701 & 0.005 \\
\hline$R^{2}$ & 0.156 & & & 0.170 & & & 0.127 & & \\
\hline
\end{tabular}

${ }^{*}$ Two-sided $P$ value; ${ }^{+}$confirmatory factor analysis (CFA) to produce a summary inflammatory index from five inflammatory markers. WBC is white blood cell count, SA is sialic acid for which the factor loading was set to 1.0 for each and both sexes, ESR is corrected erythrocyte sedimentation rate, $\alpha 1$ glob is $\alpha 1$ globulin, and $\alpha 2$ glob is alpha $\alpha 2$ globulin; ${ }^{++}$City (Naga(1)/Hiro(0)), Sex (female(1)/male(0)), ATB/10 is age at exposure (year) per 10 years, Dose is bone marrow dose (Gy), BMI/10: body mass index (weight $(\mathrm{kg}) /$ height ${ }^{2}\left(\mathrm{~m}^{2}\right)$ ) per 10, Infl. index is inflammatory index, Cancer is cancer indicator, Infl. dis. is inflammatory disease indicator, $\mathrm{DM}$ is diabetes indicator, and $\mathrm{SMK}$ is smoker indicator.

of subjects having cancer, inflammatory diseases, or diabetes was about $49 \%$ for both sexes irrespective of smoking status. The reason for such a high percentage of inflammatory diseases in Table 1 relates to our definition of inflammatory disease (Table 2) which included even mild inflammation during the examination period of 1988 to 1992 . WBC count and ESR had large sex differences, which might have been the result of a higher smoking rate in males, since WBC count is very sensitive to smoking. Table 2 shows detailed information about 23 inflammatory diseases. The diseases listed, such as osteoarthritis, cirrhosis, and infections, are considered major diseases of inflammation.

Table 3 presents information on SEM analyses for each sex and both sexes combined, as well as parameter estimates, standard errors, and two-sided $P$ values. In the M1 confirmatory factor analysis, the estimates and factor loading for SA were set to the fixed value of 1.0. The inflammatory index was constructed on the basis of this factor analysis. All factor loadings were positive and significant $(P<0.001)$, and WBC, SA, and ESR had dominant factor loadings.
The linear regression analyses for standing height M2 and for inflammatory index M3 were also shown in Table 3.

In the analysis of standing height M2, the radiation dose effect was negative and significant for both sexes combined $(P<0.001)$, for males $(P=0.005)$, and for females $(P<0.001)$. The BMI effect (per $10 \mathrm{BMI})$ was negative and significant for both sexes combined $(P<0.001)$, suggestive for males $(P=0.056)$, and significant for females $(P=$ 0.003). The multiple correlation coefficients (coefficient of determinations) were $50.9 \%$ for both sexes combined, $5.1 \%$ for males and $4.4 \%$ for females, which indicate the sex variable explains the major part of variations of height and the variables considered explain height similarly in each sex.

In the analysis of inflammatory index M3, the radiation dose effect was positive and significant for both sexes combined $(P<0.001)$, for males $(P<0.001)$, and for females $(P=0.002)$. The BMI effect (per $10 \mathrm{BMI})$ was positive and significant for both sexes combined and for females $(P<$ $0.001)$ but not significant for males $(P=0.907)$, results that are similar to those for the effect of the diabetes indicator. 
TABLE 4: Dose effect on inflammation (infl.), inflammation effect on height (ht.), indirect dose effect on height via inflammation, direct dose effect on height, total dose effect on height, and mediation proportion (MP) by sex and by age at exposure groups (standard error is the bootstrap standard error with 1,000 resamplings).

\begin{tabular}{|c|c|c|c|c|c|}
\hline \multirow{2}{*}{ Effects } & \multicolumn{5}{|c|}{ Age ATB group (year) } \\
\hline & $0-5$ & $6-17$ & $18-24$ & $0-17$ & All: $0-24$ \\
\hline & \multicolumn{5}{|c|}{ Both sexes } \\
\hline$N$ & 532 & 1,772 & 1,023 & 2,304 & 3,327 \\
\hline Dose effect on infl..$^{(a)}$ & $1.933(0.443 ;<0.001)$ & $0.917(0.294 ; 0.002)$ & $0.687(0.453 ; 0.129)$ & $1.206(0.254 ;<0.001)$ & $1.086(0.214 ;<0.001)$ \\
\hline Infl. effect on ht. ${ }^{(b)}$ & $-0.069(0.029 ; 0.018)$ & $-0.025(0.017 ; 0.141)$ & $-0.016(0.020 ; 0.430)$ & $-0.038(0.016 ; 0.018)$ & $-0.035(0.012 ; 0.004)$ \\
\hline Indirect dose effect ${ }^{(\mathfrak{c})}$ & $-0.134(0.067 ; 0.047)$ & $-0.023(0.018 ; 0.218)$ & $-0.011(0.019 ; 0.566)$ & $-0.045(0.023 ; 0.044)$ & $-0.038(0.015 ; 0.015)$ \\
\hline Direct dose effect ${ }^{(\mathrm{d})}$ & $-1.126(0.319 ;<0.001)$ & $-0.648(0.173 ;<0.001)$ & $-0.324(0.240 ; 0.176)$ & $-0.779(0.157 ;<0.001)$ & $-0.663(0.133 ;<0.001)$ \\
\hline Total dose effect ${ }^{(\mathrm{e})}$ & $-1.260(0.326 ;<0.001)$ & $-0.671(0.171 ;<0.001)$ & $-0.335(0.240 ; 0.162)$ & $-0.825(0.156 ;<0.001)$ & $-0.701(0.133 ;<0.001)$ \\
\hline \multirow[t]{2}{*}{$\mathrm{MP}^{(\mathrm{f})}(\%)(95 \% \mathrm{CI})^{(\mathrm{g})}$} & $10.6 \%(-1.1,22.4 \%)$ & $3.4 \%(-2.1,9.0 \%)$ & $3.3 \%(-8.8,15.3 \%)$ & $5.5 \%(-0.4,11.3 \%)$ & $5.4 \%(0.8,10.1 \%)$ \\
\hline & \multicolumn{5}{|c|}{ Males } \\
\hline$N$ & 227 & 704 & 212 & 931 & 1,143 \\
\hline Dose effect on infl. & $1.906(0.592 ; 0.001)$ & $1.352(0.425 ; 0.001)$ & $-0.183(1.002 ; 0.855)$ & $1.514(0.347 ;<0.001)$ & $1.311(0.317 ;<0.001)$ \\
\hline Infl. effect on ht. & $-0.003(0.060 ; 0.955)$ & $-0.036(0.031 ; 0.250)$ & $0.000(0.041 ; 0.992)$ & $-0.032(0.029 ; 0.271)$ & $-0.027(0.023 ; 0.243)$ \\
\hline Indirect dose effect & $-0.006(0.123 ; 0.958)$ & $-0.049(0.049 ; 0.314)$ & $0.000(0.043 ; 0.999)$ & $-0.049(0.048 ; 0.313)$ & $-0.036(0.033 ; 0.281)$ \\
\hline Direct dose effect & $-0.943(0.513 ; 0.066)$ & $-0.681(0.256 ; 0.008)$ & $0.034(0.583 ; 0.953)$ & $-0.725(0.226 ; 0.001)$ & $-0.622(0.221 ; 0.005)$ \\
\hline Total dose effect & $-0.949(0.509 ; 0.062)$ & $-0.729(0.252 ; 0.004)$ & $0.035(0.581 ; 0.953)$ & $-0.774(0.223 ; 0.001)$ & $-0.658(0.219 ; 0.003)$ \\
\hline \multirow[t]{2}{*}{ MP (\%) $(95 \%$ CI $)$} & $0.6 \%(-24.8,26.0 \%)$ & $6.7 \%(-7.2,20.7 \%)$ & $0.00 \%(-,-\%)$ & $6.3 \%(-6.3,19.0 \%)$ & $5.5 \%(-5.0,15.9 \%)$ \\
\hline & \multicolumn{5}{|c|}{ Females } \\
\hline$N$ & 305 & 1,068 & 811 & 1,373 & 2,184 \\
\hline Dose effect on infl. & $1.987(0.652 ; 0.002)$ & $0.457(0.409 ; 0.263)$ & $0.850(0.505 ; 0.092)$ & $0.852(0.337 ; 0.011)$ & $0.858(0.279 ; 0.002)$ \\
\hline Infl. effect on ht. & $-0.105(0.035 ; 0.003)$ & $-0.016(0.019 ; 0.391)$ & $-0.022(0.023 ; 0.338)$ & $-0.040(0.016 ; 0.013)$ & $-0.037(0.013 ; 0.006)$ \\
\hline Indirect dose effect & $-0.209(0.083 ; 0.012)$ & $-0.007(0.015 ; 0.611)$ & $-0.018(0.026 ; 0.472)$ & $-0.034(0.020 ; 0.093)$ & $-0.032(0.016 ; 0.049)$ \\
\hline Direct dose effect & $-1.311(0.395 ; 0.001)$ & $-0.601(0.223 ; 0.007)$ & $-0.404(0.255 ; 0.113)$ & $-0.832(0.206 ;<0.001)$ & $-0.696(0.165 ;<0.001)$ \\
\hline Total dose effect & $-1.520(0.414 ;<0.001)$ & $-0.609(0.222 ; 0.006)$ & $-0.422(0.255 ; 0.098)$ & $-0.866(0.208 ;<0.001)$ & $-0.728(0.166 ;<0.001)$ \\
\hline MP (\%) (95\% CI) & $13.8 \%(0.8,26.7 \%)$ & $1.1 \%(-3.7,6.0 \%)$ & $4.3 \%(-8.8,17.4 \%)$ & $3.9 \%(-1.0,8.8 \%)$ & $4.4 \%(-0.3,9.1 \%)$ \\
\hline
\end{tabular}

${ }^{(a)}$ Effect of radiation dose on inflammation (unit per Gy) with standard error (SE) and two-sided $P$ value in parenthesis; ${ }^{(b)}$ effect of inflammation index on height ( $\mathrm{cm}$ per unit) with SE and two-sided $P$ value; ${ }^{(\mathrm{c})}$ indirect dose effect on height $(\mathrm{cm} / \mathrm{Gy})=($ Dose effect on infl.) $\times($ Infl. effect on height $)$ with SE and two-sided $P$ value; ${ }^{(\mathrm{d})}$ direct dose effect on height $(\mathrm{cm} / \mathrm{Gy})$ with SE and two-sided $P$ value; ${ }^{(\mathrm{e})}$ total dose effect on height $(\mathrm{cm} / \mathrm{Gy})=\operatorname{direct}$ dose effect $(\mathrm{cm} / \mathrm{Gy})+$ indirect dose effect (cm/Gy), with SE and two-sided $P$ value; ${ }^{(\mathrm{f})}$ mediation proportion $(\mathrm{MP})=$ (indirect dose effect)/(total dose effect) $(\%) ;{ }^{(\mathrm{g})}$ approximate $95 \%$ confidence interval (CI) for the mediation proportion using the first order approximation for standard error, $\widehat{\sigma}_{\mathrm{MP}}=\sqrt{\left[\widehat{\sigma}_{T}^{2}\left(\widehat{\gamma}_{I} / \widehat{\gamma}_{T}\right)^{2}+\widehat{\sigma}_{I}^{2}\right] / \widehat{\gamma}_{T}^{2}}$, where $\widehat{\gamma}_{T}$ and $\widehat{\gamma}_{I}$ are total and indirect dose effects on height with standard errors $\widehat{\sigma}_{T}$ and $\widehat{\sigma}_{I}$, respectively.

The multiple correlation coefficients were $15.6 \%$ for both sexes, $17.0 \%$ for males, and $12.7 \%$ for females, which indicate the variables considered explain slightly more for males than for females. The correlation between BMI and diabetes variables is low (0.17).

In Table 4, direct dose effect for both sexes combined was $-1.13 \mathrm{~cm} / \mathrm{Gy}(\mathrm{SE}=0.319 ; P<0.001)$ for the $0-5$-year ATB group, $-0.65 \mathrm{~cm} / \mathrm{Gy}(\mathrm{SE}=0.173 ; P<0.001)$ for the $6-17$-year ATB group, $-0.78 \mathrm{~cm} / \mathrm{Gy}(\mathrm{SE}=0.016 ; P<0.001)$ for the $0-$ 17 -year ATB group, and $-0.66 \mathrm{~cm} / \mathrm{Gy}(\mathrm{SE}=0.013 ; P<0.001)$ for all the ages ATB group. Similar patterns for both sexes combined in significance and in tendency of reduction due to radiation were observed for each sex. The data indicate that the younger the subjects were, the larger the direct effect of radiation on height is.

In Table 4, indirect dose effect, which is a product of dose effect on the inflammation and inflammation effect on height, was significant in the $0-5$-year ATB group with $-0.13 \mathrm{~cm} / \mathrm{Gy}$ $(\mathrm{SE}=0.067 ; P=0.047)$, the $0-17$-year ATB group with
$-0.05 \mathrm{~cm} / \mathrm{Gy}(\mathrm{SE}=0.023 ; P=0.044)$, and all the ages ATB group with $-0.04 \mathrm{~cm} / \mathrm{Gy}(\mathrm{SE}=0.015 ; P=0.015)$ for both sexes combined. For males, in any of the age ATB groups, indirect dose effects were not significant $(P>0.20)$. For females, indirect dose effect was significant for the 0-5-year ATB group with $-0.21 \mathrm{~cm} / \mathrm{Gy}(\mathrm{SE}=0.083 ; P=0.012)$, suggestive for the $0-17$-year ATB group with $-0.03 \mathrm{~cm} / \mathrm{Gy}$ (SE $=0.020 ; P=0.093$ ), and significant for all the ages ATB group with $-0.03 \mathrm{~cm} / \mathrm{Gy}(\mathrm{SE}=0.016 ; P=0.049)$. For females and for both sexes combined, the youngest age ATB group had the largest indirect dose effect on height.

In Table 4, total dose effect, which is the sum of indirect dose effect and direct dose effect, was $-1.26 \mathrm{~cm} / \mathrm{Gy}(\mathrm{SE}=$ 0.326; $P<0.001)$ for the $0-5$-year ATB group, $-0.67 \mathrm{~cm} / \mathrm{Gy}$ $(\mathrm{SE}=0.171 ; P<0.001)$ for the 6-17-year ATB group, $-0.83 \mathrm{~cm} / \mathrm{Gy}(\mathrm{SE}=0.156 ; P<0.001)$ for the $0-17$-year ATB group, and $-0.70 \mathrm{~cm} / \mathrm{Gy}(\mathrm{SE}=0.133 ; P<0.001)$ for all the ages ATB group for both sexes combined. Similar patterns for both sexes combined in significance and in tendency of 
TABLE 5: Dose effect on inflammation (infl.) markers, inflammation effect on height (ht.), indirect dose effect on height via inflammation markers, direct dose effect on height, total dose effect on height, and mediation proportion (MP) by marker (standard error is the bootstrap standard error with 1,000 resamplings) for the subjects with age at exposure less than 25.

\begin{tabular}{|c|c|c|c|c|c|}
\hline \multirow{2}{*}{ Effects } & \multicolumn{5}{|c|}{ Inflammatory markers of all age ATB, $0-24$ years $(N=3,327)$} \\
\hline & $\mathrm{WBC}\left(\times 10^{2} / \mathrm{mm}^{3}\right)$ & $\mathrm{SA}(\mathrm{mg} / \mathrm{dL})$ & ESR (mm/hour) & $\alpha 1$ globulin $(\%)$ & $\alpha 2$ globulin (\%) \\
\hline & \multicolumn{5}{|c|}{ Both sexes $(N=3,327)$} \\
\hline Dose effect on infl. ${ }^{(a)}$ & $0.942(0.384 ; 0.014)$ & $0.999(0.212 ;<0.001)$ & $1.270(0.225 ;<0.001)$ & $0.052(0.018 ; 0.003)$ & $0.126(0.034 ;<0.001)$ \\
\hline Infl. effect on ht. ${ }^{(b)}$ & $-0.007(0.006 ; 0.240)$ & $-0.028(0.010 ; 0.007)$ & $-0.031(0.011 ; 0.003)$ & $0.001(0.141 ; 0.992)$ & $-0.199(0.072 ; 0.006)$ \\
\hline Indirect dose effect ${ }^{(c)}$ & $-0.006(0.006 ; 0.318)$ & $-0.028(0.012 ; 0.021)$ & $-0.039(0.015 ; 0.009)$ & $0.000(0.008 ; 0.992)$ & $-0.025(0.012 ; 0.030)$ \\
\hline Direct dose effect ${ }^{(\mathrm{d})}$ & $-0.697(0.133 ;<0.001)$ & $-0.672(0.133 ;<0.001)$ & $-0.661(0.134 ;<0.001)$ & $-0.702(0.134 ;<0.001)$ & $-0.676(0.133 ;<0.001)$ \\
\hline Total dose effect ${ }^{(\mathrm{e})}$ & $-0.703(0.133 ;<0.001)$ & $-0.701(0.133 ;<0.001)$ & $-0.701(0.133 ;<0.001)$ & $-0.702(0.133 ;<0.001)$ & $-0.701(0.133 ;<0.001)$ \\
\hline \multirow[t]{2}{*}{$\mathrm{MP}^{(\mathrm{f})}(\%)(95 \% \mathrm{CI})^{(\mathrm{g})}$} & $0.9 \%(-0.8,2.6 \%)$ & $4.0 \%(0.3,7.7 \%)$ & $5.6 \%(0.9,10.2 \%)$ & $0.0 \%(-2.2,2.2 \%)$ & $3.6 \%(0.0,7.2 \%)$ \\
\hline & \multicolumn{5}{|c|}{ Males $(N=1,143)$} \\
\hline Dose effect on infl. & $.735(0.675 ; 0.276)$ & $1.188(0.333 ;<0.001)$ & $1.564(0.324 ;<0.001)$ & $0.079(0.027 ; 0.0$ & 0.128 \\
\hline Infl. effect on ht. & $-0.005(0.009 ; 0.592)$ & $-0.020(0.020 ; 0.327)$ & $-0.020(0.023 ; 0.383)$ & $-0.180(0.263 ; 0.493)$ & $.157)$ \\
\hline Indirect dose effect & $-0.004(0.010 ; 0.727)$ & $-0.024(0.027 ; 0.375)$ & $-0.031(0.037 ; 0.403)$ & $-0.014(0.023 ; 0.530)$ & $-0.023(0.019$ \\
\hline Direct dose effect & $-0.655(0.219 ; 0.003)$ & $-0.634(0.220 ; 0.004)$ & $-0.625(0.224 ; 0.005)$ & $-0.644(0.220 ; 0.003)$ & $-0.636(0.219 ; 0.004)$ \\
\hline Total dose effect & $-0.659(0.219 ; 0.003)$ & $-0.657(0.219 ; 0.003)$ & $-0.656(0.219 ; 0.003)$ & $-0.658(0.219 ; 0.003)$ & $-0.659(0.219 ; 0.003)$ \\
\hline \multirow[t]{2}{*}{ MP (\%) (95\% CI) } & & & $4.7 \%(-6.8,16.2 \%)$ & & \\
\hline & \multicolumn{5}{|c|}{ Females $(N=2,184)$} \\
\hline Dose effect on infl. & $1.035(0.433 ; 0.017)$ & $0.787(0.275 ; 0.004)$ & $1.002(0.308 ; 0.001)$ & $0.030(0.023 ; 0.178)$ & $0.116(0.044 ; 0.009)$ \\
\hline Infl. effect on ht. & $-0.008(0.008 ; 0.301)$ & $-0.032(0.012 ; 0.007)$ & $-0.034(0.011 ; 0.003)$ & $0.106(0.176 ; 0.548)$ & $-0.208(0.084 ; 0.013)$ \\
\hline Indirect dose effect & $-0.009(0.010 ; 0.385)$ & $-0.026(0.013 ; 0.058)$ & $-0.034(0.016 ; 0.029)$ & $0.003(0.007 ; 0.649)$ & $-0.024(0.014 ; 0.077)$ \\
\hline Direct dose effect & $-0.724(0.166 ;<0.001)$ & $-0.703(0.165 ;<0.001)$ & $-0.696(0.166 ;<0.001)$ & $-0.736(0.166 ;<0.001)$ & $-0.705(0.166 ;<0.001)$ \\
\hline Total dose effect & $-0.732(0.166 ;<0.001)$ & $-0.728(0.166 ;<0.001)$ & $-0.731(0.166 ;<0.001)$ & $-0.733(0.166 ;<0.001)$ & $-0.729(0.166 ;<0.001)$ \\
\hline MP (\%) (95\% CI) & $1.2 \%(-1.5,4.0 \%)$ & $3.6 \%(-4.7,12.1 \%)$ & $4.7 \%(-0.1,9.4 \%)$ & $-0.4 \%(-1.5,2.3 \%)$ & $3.3 \%(-0.7,7.3 \%)$ \\
\hline
\end{tabular}

${ }^{(a)}$ Effect of radiation dose on inflammation marker (unit per Gy) with standard error (SE) and two-sided $P$ value in parenthesis; ${ }^{(b)}$ effect of inflammation marker on height (cm per unit) with SE and two-sided $P$ value; ${ }^{(c)}$ indirect dose effect on height $(\mathrm{cm} / \mathrm{Gy})=($ Dose effect on infl. $) \times($ Infl. effect on height $)$ with SE and two-sided $P$ value; ${ }^{(d)}$ direct dose effect on height $(\mathrm{cm} / \mathrm{Gy})$ with SE and two-sided $P$ value; ${ }^{(e)}$ total dose effect on height $(\mathrm{cm} / \mathrm{Gy})=\operatorname{direct}$ dose effect $(\mathrm{cm} / \mathrm{Gy})+$ indirect dose effect (cm/Gy), with SE and two-sided $P$ value; ${ }^{(\mathrm{f})}$ mediation proportion (MP) = (indirect dose effect)/(total dose effect) (\%); ${ }^{(\mathrm{g})}$ approximate $95 \%$ confidence interval (CI) for the mediation proportion using the first order approximation for standard error, $\widehat{\sigma}_{\mathrm{MP}}=\sqrt{\left[\widehat{\sigma}_{T}^{2}\left(\widehat{\gamma}_{I} / \widehat{\gamma}_{T}\right)^{2}+\widehat{\sigma}_{I}^{2}\right] / \widehat{\gamma}_{T}^{2}}$, where $\widehat{\gamma}_{T}$ and $\widehat{\gamma}_{I}$ are total and indirect dose effects on height with standard errors $\widehat{\sigma}_{T}$ and $\widehat{\sigma}_{I}$, respectively.

reduction due to radiation were also observed for each sex. The total dose effect on height was greater in the younger age ATB groups similar to the pattern shown in Nakashima et al. [2].

In Table 4, the mediation proportion of indirect dose effect via inflammation was obtained by dividing the indirect dose effect by the total dose effect. In the 0-5-year ATB group, the MPs due to inflammation were $0.6 \%, 13.8 \%$, and $10.6 \%$ for males, for females, and for both sex combined, respectively. In the 0-17-year ATB group and the 0-24-year ATB group, the MPs due to inflammation were between $4 \%$ and $6 \%$ for males, for females, and both sexes combined.

In Table 5, the results of SEM analyses for each inflammatory marker for all ages of 0-24-year ATB are presented. The direct and total effects were significant $(P<0.005)$ for all inflammatory markers for males, for females, and for both sexes combined. The indirect dose effects were significant for both sexes combined via SA $(P=0.021)$, ESR $(P=0.009)$, and $\alpha 2$ globulin $(P=0.030)$. The indirect effect for males was not significant via any of the inflammatory markers. The indirect effect for females was significant via SA $(P=0.058)$, ESR $(P=0.029)$, and $\alpha 2$ globulin $(P=0.077)$. The MPs of indirect effect via SA, ESR, and $\alpha 2$ globulin were from $3 \%$ to $6 \%$ in both sexes combined and in each sex.

\section{Discussion}

When the log-transformed variable for inflammation was used for analyses, in some of the cases, computation did not converge, although, in general, log-transformation results in larger effect and more significant result. Thus we made the analyses using original inflammation data without logtransformation. When we calculate the standard errors (SEs) for mediation proportions (MPs), we used asymptotic standard errors for the construction of the confidence intervals (CIs) dropping the covariance between total and indirect effects, which results in possibly wider (or conservative) CIs due to possibly positive correlation between total and indirect effects.

Our results are consistent with those obtained by Neriishi et al. [12], which used principal component analysis instead of factor analysis to construct the inflammatory index from the same five inflammatory markers. The MPs were slightly larger in the analysis by Neriishi et al. [12] than in 
the current analysis, which may be because Neriishi et al. [12] did not adjust the inflammation index for risk factors such as inflammatory diseases and BMI. It is also interesting that BMI in our study was inversely related to standing height for both sexes combined and indicate enhanced inflammation especially in females, the latter being similar to the diabetes indicator effect.

Among the five inflammatory markers, SA, corrected ESR, and $\alpha 2$ globulin had significant indirect dose effects as indicated in Table 5. SA and the globulins were highly specific to inflammation, and corrected ESR and WBC were relatively nonspecific to inflammation. However, the reason for the significant result of indirect dose effect via each of these three markers in Table 5, especially for females, is unclear. The MP of $\alpha 1$ globulin seems to be lower than other markers. This might be due to influence of acute inflammation on this marker [22].

As an inflammation surrogate, we used the inflammatory index constructed with SEM analysis from the five inflammatory markers measured between 1988 and 1992. More specifically, in Figure 1 of our SEM model, we can assume a hypothetical inflammatory index $Z$ when the subjects were young, which is different from $Y$. The two indexes $Y$ and $Z$ have standard deviations $\sigma_{Y}$ and $\sigma_{Z}$, respectively, and are correlated with each other with correlation coefficient, $1>$ $\rho>0$, which cannot be large due to the long time lag between two measurements $Y$ and $Z$. If we assume that $\alpha^{*}$ is the approximate linear dose effect on the inflammatory index when subjects were young and $\beta^{*}$ is the approximate linear effect of inflammatory index on height when young, we can then define $\alpha=\alpha^{*} \delta_{1}$ and $\beta=\delta_{2} \beta^{*}$, where $\delta_{1}=\rho \sigma_{Y} / \sigma_{Z}$ is the regression coefficient of $Y$ on $Z$ and $\delta_{2}=\rho \sigma_{Z} / \sigma_{Y}$ is the regression coefficient of $Z$ on $Y$. The indirect radiation effect in the period of 1988 to 1992 can be approximately written as $\alpha \beta=\rho^{2} \alpha^{*} \beta^{*}<\alpha^{*} \beta^{*}$, where $\alpha^{*} \beta^{*}$ is the indirect mediation effect when subjects were young via inflammatory index $Z$. Since $\rho^{2}$ is positive and less than one, it should be noted that the indirect radiation dose effect on standing height could very well be seriously underestimated using inflammatory index from 1986 to 1992.

Our results indicate that, in the 0-5-year ATB group, negative indirect radiation effect on standing height was significant except for males. The reason for this nonsignificance among males in the 0-5-year ATB group is unclear. Note that, among inflammatory diseases listed in Table 2, some might have been experienced in childhood but the age at disease development is unknown. Other events, such as smoking, cancer, and diabetes, were likely not experienced in childhood, and the information that the smoking rate was $54 \%$ for males and only $10 \%$ for females should also be noted. Smoking has a significant impact on the inflammation, especially in terms of WBC counts. We changed the cutoff age of 6.0 years to 4.0 years and 10.0 years and then reanalyzed the data for the 0 - to 4-year ATB group and 0 - to 10 year ATB group, both including and not including smokers. In all cases, the mediation effect by inflammation was still not significant for males but was significant for females and for both sexes combined. We therefore concluded that a significant mediation effect via inflammation exists for females exposed when young.

The data indicate that the younger the exposure to radiation, the larger the mediation effect of inflammation. Such inflammation can be interpreted to indicate that when exposure to radiation occurs earlier in childhood, radiation dose effects on inflammation and standing height last until maturation of growth, or an age of about 20 years, and thus the period of exposure to enhanced inflammation is longer. The results for indirect dose effects were larger for the earlier age ATB groups.

The radiation dose effect includes social or health status differences along the distance from hypocenter. However, the dose effect along ages would be a true biological effect due to the possible independence between distance from hypocenter and age at exposure. The socioeconomic status effect was possibly but not perfectly excluded from the standing height by inclusion of BMI in the regression analysis M2 and the radiation dose effect would be thought as pure biological effect. The selection bias also exists in our sample, because our sample was the people who survived till 1958 when AHS cohort was established and further survived till this study period, 1988-1992.

We tried to prove two issues for the indirect radiation effect on height via the inflammation; that is, the indirect effect is statistically significant and meaningful in physical science. Although the first point was successful except for male, we failed to prove the second point. In fact, even if the significant MP is $10-20 \%$, it would be meaningless in terms of physical science because, for example, when the total effect was a reduction of $2 \mathrm{~cm} / \mathrm{Gy}$, although actually Table 3 indicates reduction due to radiation was much smaller than this, the indirect effect would result in a reduction of 2$4 \mathrm{~mm} / \mathrm{Gy}$ with MP of $10-20 \%$, which would be medically meaningless and within the confidence interval. However, the following points should be mentioned. First, although we used the five inflammatory markers, these would not be the full proxy of the inflammation of the living body. Second, nevertheless, the existence of the significant indirect effect was proved in irradiated human, supporting experimental evidence of the bystander effect of the inflammation $[23,24]$. Moreover, since inflammatory factors, such as Reactive Oxygen species [25], FasL, and TNF-alpha [24], have been proved as mediators and since nonsteroidal anti-inflammation drug treatment reduced such damage in vivo [26], our findings provide basis for clinical use of such drugs for prevention for radiation exposed human.

There are several limitations to the current study. Selection biases exist due to deaths of survivors during the period from the beginning of the AHS to the time of measurements. Bias may also arise due to missing data; the current study sample $(3,327$ subjects) has $19 \%$ missing data from the original study sample (4,076 subjects). We assumed that these biases do not substantially affect the results. We also assumed that inflammation persists until adulthood, which is partly supported by the literature [7-11] as stated in the Introduction. Kaufman et al. [27] criticized the current method of SEM $[14,16]$ in regard to the assumption that the total effect can be partitioned into direct and indirect effects in 
additive fashion, which has a straightforward interpretation. Although checking this assumption is difficult, we assumed that it is true in the first order linear approximation and this linear approximation does not greatly lower the estimation efficiency, that is, does not substantially enlarge the standard errors of the estimates.

In conclusion, our results indicate that, in the 0-5-year ATB group, negative indirect radiation effect on standing height was significant except for males even after adjustment was made for such inflammatory factors as smoking, cancer, inflammatory disease, BMI, and diabetes. The indirect effect among age 0 to 5 was a loss of $-0.13 \mathrm{~cm}(\mathrm{SE}=0.067 ; P=$ $0.047)$ per Gy. In this age group, the indirect effect was larger and statistically significant for females with $-0.13 \mathrm{~cm}$ per Gy ( $\mathrm{SE}=0.083 ; P=0.012)$ while the indirect effects was smaller and not statistically significant among males with $-0.01 \mathrm{~cm}$ per Gy $(\mathrm{SE}=0.123 ; P=0.958)$. The fraction attributable to inflammation in reduction of height due to radiation is small, but the MP of radiation dose effect on height via inflammation was approximately $4 \%$ to $6 \%$ for both sexes. By individual inflammatory marker, indirect dose effects in all ages were significant via sialic acid, corrected ESR, and $\alpha 2$ globulin for both sexes combined $(P<0.03)$ and for females $(P<0.08)$ and the MPs of the indirect effect via these three inflammatory markers ranged from $3 \%$ to $6 \%$. One reason why the mediation effect was not significant in males may have been their higher smoking rate. These findings suggest the existence of significant indirect effects of A-bomb radiation on height via persistent subclinical inflammation for young survivors in addition to a significant direct effect of A-bomb radiation on height. The indirect effects were likely to be seriously underestimated due to the time lag between the A-bombings and the inflammatory measurements.

\section{Disclaimer}

The views of the authors do not necessarily reflect those of the two governments.

\section{Conflict of Interests}

The authors declare that there is no conflict of interests regarding the publication of this paper.

\section{Acknowledgments}

The authors thank Ms. Sachiyo Funamoto for her assistance in data preparation, Mr Jeffrey Hart, Drs John Cologne and Harry Cullings for their editorial help, and anonymous referees for their helpful comments. RERF is a private, nonprofit foundation funded by the Japanese Ministry of Health, Labour and Welfare (MHLW) and the U.S. Department of Energy (DOE), the latter partly through DOE Award DEHS0000031 to the National Academy of Sciences which supported Wan-Ling Hsu. This paper was supported by RERF research protocol no. 2-75.

\section{References}

[1] M. Otake, Y. Fujikoshi, S. Funamoto, and W. J. Schull, "Evidence of radiation-induced reduction of height and body weight from repeated measurements of adults exposed in childhood to the atomic bombs," Radiation Research, vol. 140, no. 1, pp. 112-122, 1994.

[2] E. Nakashima, S. Fujiwara, and S. Funamoto, "Effect of radiation dose on the height of atomic bomb survivors: a longitudinal study," Radiation Research, vol. 158, no. 3, pp. 346-351, 2002.

[3] J. L. Belsky, K. Tachikawa, and S. Jablon, "ABCC-JNIH Adult health study, report 5. Results of the first five examination cycles, Hiroshima-Nagasaki 1958-1968," Yale Journal of Biology and Medicine, vol. 46, pp. 284-296, 1973.

[4] H. Sawada, K. Kodama, Y. Shimizu, and H. Kato, "Adult health study report 6. Results of six examination cycles, 1968-80, Hiroshima and Nagasaki," Tech. Rep. 3-86, Radiation Effects Research Foundation, 1987.

[5] K. Neriishi, E. Nakashima, and R. R. Delongchamps, "Persistent subclinical inflammation among A-bomb survivors," International Journal of Radiation Biology, vol. 77, no. 4, pp. 475-482, 2001.

[6] W.-L. Hsu, Y. Tatsukawa, K. Neriishi, M. Yamada, J. B. Cologne, and S. Fujiwara, "Longitudinal trends of total white blood cell and differential white blood cell counts of atomic bomb survivors," Journal of Radiation Research, vol. 51, no. 4, pp. 431439, 2010.

[7] S. Gaspari, M. L. Marcovecchio, L. Breda, and F. Chiarelli, "Growth in juvenile idiopathic arthritis: the role of inflammation," Clinical and Experimental Rheumatology, vol. 29, no. 1, pp. 104-110, 2011.

[8] F. de Benedetti, T. Alonzi, A. Moretta et al., "Interleukin 6 causes growth impairment in transgenic mice through a decrease in insulin-like growth factor-I. A model for stunted growth in children with chronic inflammation," Journal of Clinical Investigation, vol. 99, no. 4, pp. 643-650, 1997.

[9] V. H. Heemskerk, M. A. R. C. Daemen, and W. A. Buurman, "Insulin-like growth factor-1 (IGF-1) and growth hormone (GH) in immunity and inflammation," Cytokine and Growth Factor Reviews, vol. 10, no. 1, pp. 5-14, 1999.

[10] P. C. Owens, K. L. Gatford, P. E. Walton, W. Morley, and R. G. Campbel, "The relationship between endogenous insulin-like growth factors and growth in pigs," Journal of Animal Science, vol. 77, no. 8, pp. 2098-2103, 1999.

[11] R. J. Ferry Jr., R. W. Cerri, and P. Cohen, "Insulin-like growth factor binding proteins: new proteins, new functions," Hormone Research, vol. 51, no. 2, pp. 53-67, 1999.

[12] K. Neriishi, E. Nakashima, and G. Suzuki, "Relationship between growth retardation and inflammation among A-bomb survivors," in Proceedings of the Meeting on Late Effect A-Bomb Radiation, vol. 77, pp. 227-230, 2002.

[13] I. T. Jolliffe, Principal Component Analysis, Springer Series in Statistics, Springer, New York, NY, USA, 1986.

[14] S. Ditlevsen, U. Christensen, J. Lynch, M. T. Damsgaard, and N. Keiding, "The mediation proportion: a structural equation approach for estimating the proportion of exposure effect on outcome explained by an intermediate variable," Epidemiology, vol. 16, no. 1, pp. 114-120, 2005.

[15] F. Emanuela, M. Grazia, D. R. Marco, L. Maria Paola, F. Giorgio, and B. Marco, "Inflammation as a link between obesity and metabolic syndrome," Journal of Nutrition and Metabolism, vol. 2012, Article ID 476380, 7 pages, 2012. 
[16] L. K. Muthen and B. O. Muthen, M-Plus Package, Version 6, Muthén \& Muthén, Los Angeles, Calif, USA, 2010.

[17] G. W. Beebe, H. Fujisawa, and M. Yamasaki, "ABCC-JNIH adult health study reference paper," Tech. Rep. 10-60, Radiation Effects Research Foundation, 1960.

[18] K. Yokoro, "A review of forty-five years study of Hiroshima and Nagasaki atomic bomb survivors," Journal of Radiation Research, vol. 32, supplement 1, pp. 15-442, 1991.

[19] D. L. Preston, E. Ron, S. Tokuoka et al., "Solid cancer incidence in atomic bomb survivors: 1958-1998," Radiation Research, vol. 168, no. 1, pp. 1-64, 2007.

[20] R. W. Young and G. D. Kerr, Eds., Reassessment of the Atomic Bomb Radiation Dosimetry for Hiroshima and Nagasaki, Dosimetry System 2002. Report of the Joint US-Japan Working Group, Radiation Effects Research Foundation, Hiroshima, Japan, 2005.

[21] D. A. Pierce, D. O. Stram, and M. Vaeth, "Allowing for random errors in radiation dose estimates for the atomic bomb survivor data," Radiation Research, vol. 123, no. 3, pp. 275-284, 1990.

[22] I. Kushner and Z. A. Mackiewics, "The acute phase response: an overview," in Acute-Phaseglycoprotains: Molecular Biology, Biochemistry and Clinical Application, pp. 3-19, CRC Press, New York, NY, USA, 1993.

[23] S. A. Lorimore, P. J. Coates, G. E. Scobie, G. Milne, and E. G. Wright, "Inflammatory-type responses after exposure to ionizing radiation in vivo: a mechanism for radiation-induced bystander effects?" Oncogene, vol. 20, no. 48, pp. 7085-7095, 2001.

[24] S. Rastogi, P. J. Coates, S. A. Lorimore, and E. G. Wright, "Bystander-type effects mediated by long-lived inflammatory signaling in irradiated bone marrow," Radiation Research, vol. 177, no. 3, pp. 244-250, 2012.

[25] C. Shao, Y. Furusawa, Y. Kobayashi, T. Funayama, and S. Wada, "Bystander effect induced by counted high-LET particles in confluent human fibroblasts: a mechanistic study," The FASEB Journal, vol. 17, no. 11, pp. 1422-1427, 2003.

[26] D. Mukherjee, P. J. Coates, S. A. Lorimore, and E. G. Wright, "Responses to ionizing radiation mediated by inflammatory mechanisms," The Journal of Pathology, vol. 232, no. 3, pp. 289299, 2014.

[27] J. S. Kaufman, R. F. MacLehose, S. Kaufman, and S. Greenland, "The mediation proportion," Epidemiology, vol. 16, no. 5, p. 710, 2005. 


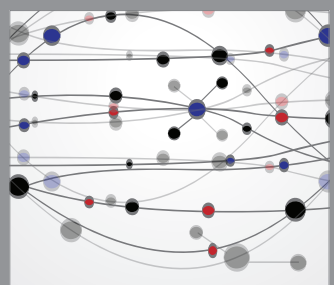

The Scientific World Journal
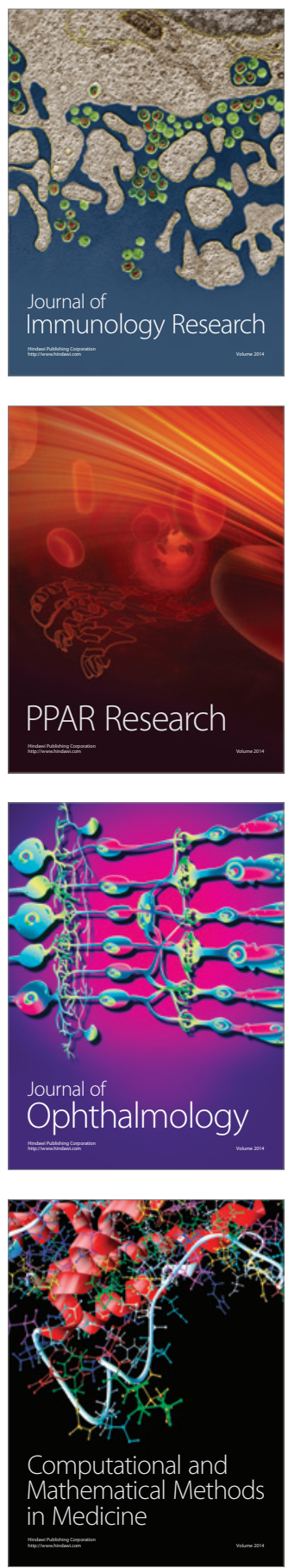

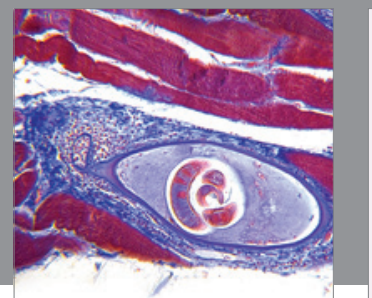

Gastroenterology

Research and Practice
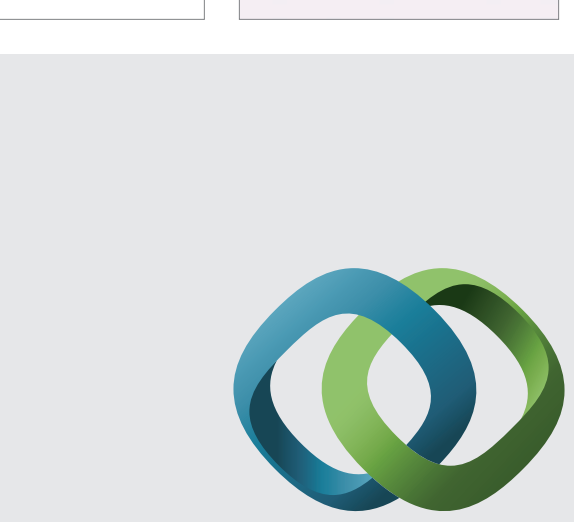

\section{Hindawi}

Submit your manuscripts at

http://www.hindawi.com
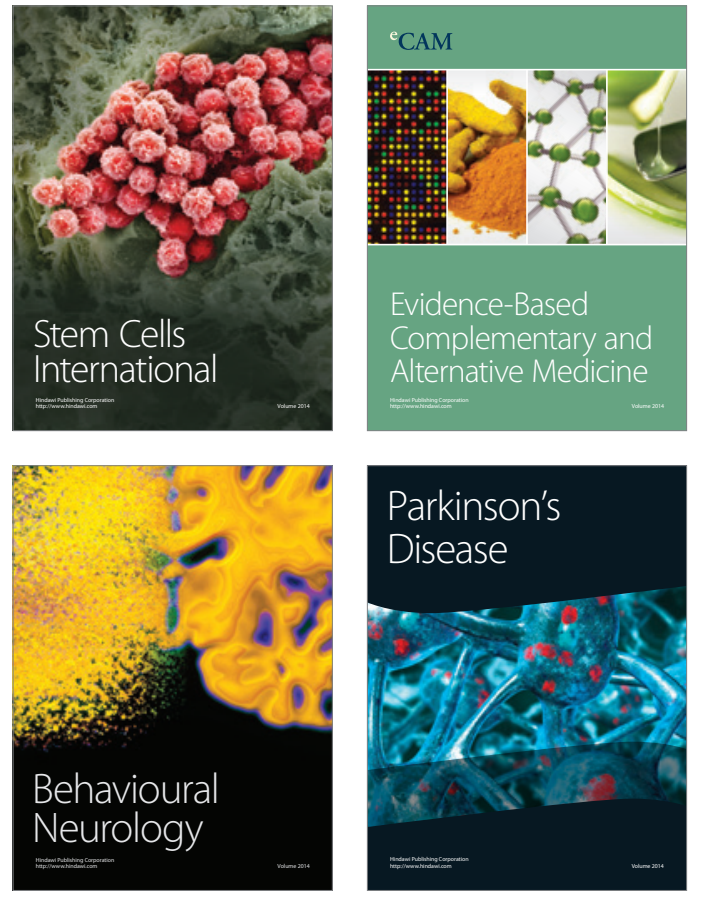
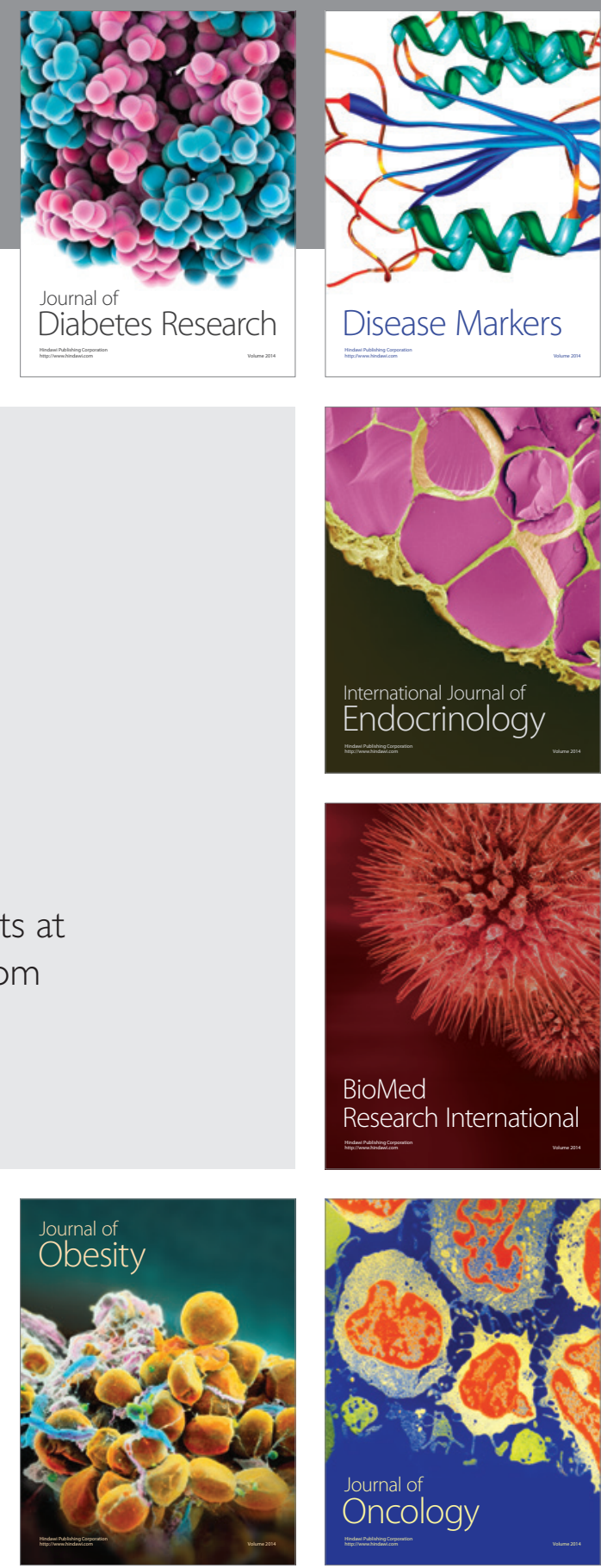

Disease Markers
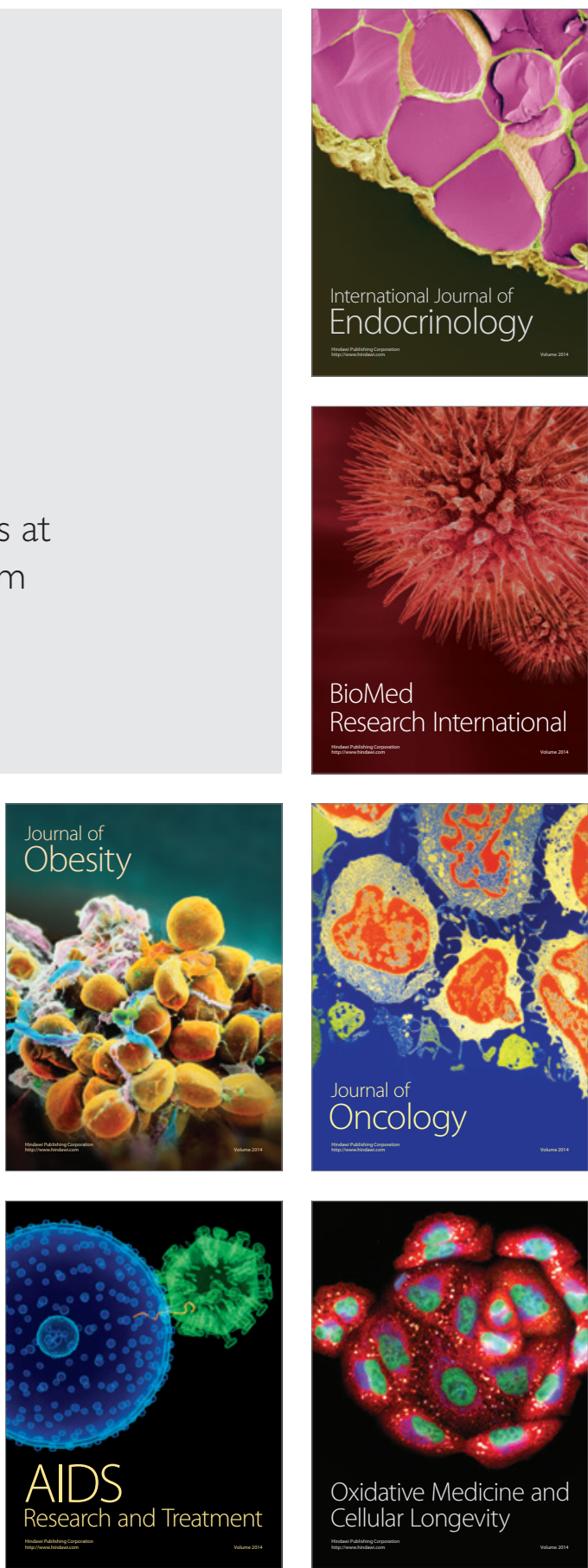